\title{
LONG-TERM EFFECTS OF EARLY PULSE INTRAVENOUS METHYLPREDNISOLONE OR INTRAVENOUS IMMUNOGLOBULIN IN ADULT PATIENTS WITH DERMATOMYOSITIS AND POLYMYOSITIS: A LARGE RETROSPECTIVE COHORT STUDY
}

\author{
Leonardo Santos Hoff ${ }^{1}$, Fernando Henrique Carlos de Souza ${ }^{1}$, Renata Miossi ${ }^{1}$, Samuel Katsuyuki Shinjo ${ }^{1, \star}$ \\ 1. Faculdade de Medicina FMUSP, Universidade de São Paulo, São Paulo (SP), Brazil. \\ *Corresponding author: samuel.shinjo@usp.br
}

\section{BACKGROUND}

Dermatomyositis (DM) and polymyositis (PM) are systemic autoimmune myopathies characterized by chronic muscle inflammation and systemic symptoms. The optimal initial treatment of DM and PM remains controversial given their rarity, the heterogeneous presentation of the diseases, and the few randomized controlled trials published so far. Evidence for the efficacy of pulse intravenous methylprednisolone (IVMP) or intravenous immunoglobulin (IVIg) comes from observational or experimental studies with a small number of patients, and their long-term effects on different disease outcomes remain undetermined. This study aims to evaluate the long-term effects of early pulse IVMP or IVIg in a large cohort of patients with DM and PM.

\section{METHODS}

This is a retrospective single-center cohort study of adult patients with DM and PM classified with the Bohan and Peter criteria and followed for up to four years from 2001 to 2017. Pulse IVMP or IVIg received as an add-on to the usual therapy during the first year of diagnosis were considered early interventions. We used Cox proportional hazard regression models to estimate hazard ratios (HR) with a 95\% confidence interval (Cl) for three outcomes: complete clinical response, corticosteroid discontinuation, and mortality. The models were adjusted for clinical, laboratory, and treatment covariates.

\section{RESULTS}

A total of 204 patients were included and categorized into four initial treatment groups: pulse IVMP $(n=46)$, pulse IVMP+IVIg $(n=55)$, IVIg $(n=10)$, and without IVMP or IVIg $(n=93)$. Patients submitted to pulse IVMP or IVIg had more dysphagia, severe proximal weakness, and infections, as well as higher creatine phosphokinase serum levels. The early pulse IVMP and pulse IVMP+IVIg groups had a higher HR for complete clinical response in the multivariate model: $\mathrm{HR}=1.56,95 \% \mathrm{Cl}=1.05-2.33$, $p=0.029$, and $\mathrm{HR}=1.58,95 \% \mathrm{Cl}=1.02-2.45, p=0.041$, respectively. Only the group of pulse IVMP+IVIg had a significant association with corticosteroid discontinuation in the multivariate analysis: $\mathrm{HR}=1.65,95 \% \mathrm{Cl}=1.02-2.68, \mathrm{p}=0.043$. Neither early pulse IVMP nor IVIg influenced mortality.

\section{CONCLUSION}

Despite having a more severe disease profile, patients with DM and PM submitted to pulse IVMP or pulse IVMP+IVIg during the first year of diagnosis had a higher HR for complete clinical response, whereas the combination of pulse IVMP+IVIg had an association with corticosteroid discontinuation. Further long-term studies are warranted to confirm these benefits of early pulse IVMP and IVIg on patients with DM and PM.

\section{KEYWORDS}

Dermatomyositis, Polymyositis, Intravenous immunoglobulin, Methylprednisolone, Idiopathic inflammatory myopathies. 